\title{
An Algorithm for Fitting Local Membrane Parameters to an Action Potential Duration Map in a Tissue with Electrotonic Interactions
}

\author{
Angelina Drahi ${ }^{1,2}$, Akshay Kota Aswath $\operatorname{Kumar}^{1,3}$, Vincent Jacquemet ${ }^{1,3}$ \\ ${ }^{1}$ Université de Montréal, Montréal, Canada \\ ${ }^{2}$ École Polytechnique Fédérale de Lausanne, Lausanne, Switzerland \\ ${ }^{3}$ Hôpital du Sacré-Cœur de Montréal, Montréal, Canada
}

\begin{abstract}
Repolarization gradients contribute to arrhythmogenicity. They can be introduced in computer models of cardiac tissue by locally adjusting an intrinsic parameter of the membrane model. Electronic coupling, however, modulates the dispersion of action potential duration (APD).

We developed an algorithm based on a modified Newton method to iteratively adjust the spatial distribution of a membrane parameter in order to reproduce a given APD map in a coupled tissue. The method was applied to an atrial model with randomly generated APD maps with controllable maximum APD gradient. The adjustable local parameter was the conductance of the $I_{\mathrm{K}, \mathrm{ACh}}$ current. Convergence was found to be faster when the maximal gradient was less steep and when tissue conductivity was reduced.

This algorithm provides a tool to automatically generate arrhythmogenic substrates with controllable repolarization gradients and possibly incorporate experimental APD maps into computer models.
\end{abstract}

\section{Introduction}

The action potential duration (APD) of a cardiac cell estimates the duration of its refractory period. APD dispersion may result from regional electrophysiological differences possibly aggravated by the remodeling induced by successive episodes of arrhythmia. Steep gradients in APD promote conduction blocks and functional reentries. However, in a tissue where cells are coupled through gap junctions, electrotonic currents tend to reduce the differences in APD between neighboring cells [1-3]. This means that the distribution of APD measured in a tissue may significantly differ from the distribution of intrinsic APD that would have been observed if the cells were uncoupled.

In computer models of cardiac arrhythmia, APD dispersion is introduced by designing a spatial profile of intrinsic properties of cardiac cells. Typically, the spatial distribution of a parameter is used as an input of the model. The question arises whether that parameter distribution can be determined from the APD map in the coupled tissue. Defauw et al. proposed a Gaussian Green's function model and a deconvolution approach to address this problem [4]. We solved analytically this problem in a toy model assuming that the APD gradient was not too steep [5]. Here, we implemented and validated a computational approach in which the parameter distribution is iteratively updated to reproduce a target APD map.

\section{Methods}

\subsection{Problem statement}

In the framework of a monodomain model of cardiac tissue, let us consider that the membrane model depends on a local parameter $k$ that can vary within the range $\left[k_{\min }, k_{\max }\right]$. This parameter could be an ion channel conductance, an ionic concentration, or a normalized parameter describing the transition between normal and diseased tissue, but is assumed not to affect intercellular coupling (gap junction conductances). After spatial discretization, tissue configuration is described by a vector $\mathbf{k}$ whose size is the number of nodes in the mesh.

Due to the non-uniform distribution of $k$, the simulated APD map is also non-uniform. The forward problem consists in computing by simulation the APD map (a) as a function of $\mathbf{k}$

$$
\mathbf{a}=\mathbf{a}_{\text {forw }}(\mathbf{k} ; \mathbf{G}) \text {. }
$$

Because of electrotonicity, APD distribution depends not only on $\mathbf{k}$ but also on intercellular coupling matrix $\mathbf{G}$.

Assuming that the coupling is known, the inverse problem consists in recovering the parameter distribution $\mathbf{k}$ that would reproduce a given APD map $\mathbf{a}_{\text {target }}$

$$
\mathbf{k}=\mathbf{a}_{\text {forw }}^{-1}\left(\mathbf{a}_{\text {target }} ; \mathbf{G}\right) \text {, }
$$

provided that the solution exists and is unique, i.e. $\mathbf{a}_{\text {forw }}$ is invertible. Uniqueness of the solution has been proved 
in a simple analytical model [5] and is also guaranteed (although not easy to estimate) if the APD map in the coupled system can be written as the convolution of the intrinsic APD map with a spatial (e.g. Gaussian) filter [4].

\subsection{Parameter identification}

The inverse problem is equivalent to solving the equation $\mathbf{a}_{\text {forw }}(\mathbf{k} ; \mathbf{G})-\mathbf{a}_{\text {target }}=\mathbf{0}$. Our approach will rely on the fact that the problem is easily solved when cells are uncoupled ( $\mathbf{G}=\mathbf{0}$; see next subsection). A first approximation $\mathbf{k}^{(0)}$ is obtained by neglecting electrotonicity:

$$
\mathbf{k}^{(0)}=\mathbf{a}_{\text {forw }}^{-1}\left(\mathbf{a}_{\text {target }} ; \mathbf{0}\right) .
$$

Then, at iteration $n$, the parameter profile is updated using the Newton-Raphson formula

$$
\begin{aligned}
\mathbf{k}^{(n+1)}=\mathbf{k}^{(n)}- & \left(\mathbf{D} \mathbf{a}_{\text {forw }}\left(\mathbf{k}^{(n)} ; \mathbf{0}\right)\right)^{-1} \\
& \times\left(\mathbf{a}_{\text {forw }}\left(\mathbf{k}^{(n)} ; \mathbf{G}\right)-\mathbf{a}_{\text {target }}\right),
\end{aligned}
$$

where the Jacobian $\mathbf{D a}_{\text {forw }}\left(\mathbf{k}^{(n)} ; \mathbf{G}\right)$ has been approximated by the (diagonal) Jacobian in the uncoupled tissue $\mathbf{D a}_{\text {forw }}\left(\mathbf{k}^{(n)} ; \mathbf{0}\right)$ to avoid expensive computations. The iteration process stops when the 99th percentile of the absolute error $\left|\mathbf{a}_{\text {forw }}\left(\mathbf{k}^{(n)}\right)-\mathbf{a}_{\text {target }}\right|$ falls below a tolerance, typically $1 \mathrm{~ms}$. This choice of termination criterion reduces overfitting since no regularization constraint on $\mathrm{k}$ was used.

\subsection{Solving the uncoupled case}

As a preprocessing step, the relation $a=\alpha(k)$ between the parameter $k$ and the $\operatorname{APD}(a)$ was studied in an isolated cell. The function $\alpha$ was evaluated (using simulations) at 8 equally-spaced points in the interval $\left[k_{\min }, k_{\max }\right]$. The number of points was then iteratively increased until the maximal error between spline interpolation based on the previous iteration and the new computed data points fell below a threshold, typically $0.5 \mathrm{~ms}$. This provided a piecewise polynomial interpolation for the function $\alpha(k)$. The monotonicity of $\alpha(k)$ was checked using the coefficient of the polynomials. Spline interpolation on the same data points (reflected across the diagonal) was used to compute the inverse function $k=\alpha^{-1}(a)$. The derivative $\alpha^{\prime}(k)$ was obtained by analytically derivating the piece-wise polynomial in each of its segments. To avoid out-of-bound errors, when the argument of the function is out of the domain or the range of $\alpha$, the value at the bound is returned.

With these notations, we have:

$$
\begin{aligned}
\mathbf{a}_{\text {forw }}^{-1}\left(\mathbf{a}_{\text {target }} ; \mathbf{0}\right) & =\alpha^{-1}\left(\mathbf{a}_{\text {target }}\right) \\
\left(\mathbf{D a}_{\text {forw }}(\mathbf{k} ; \mathbf{0})\right)^{-1} & =\operatorname{diag}\left(\alpha^{\prime}(\mathbf{k})\right)^{-1},
\end{aligned}
$$
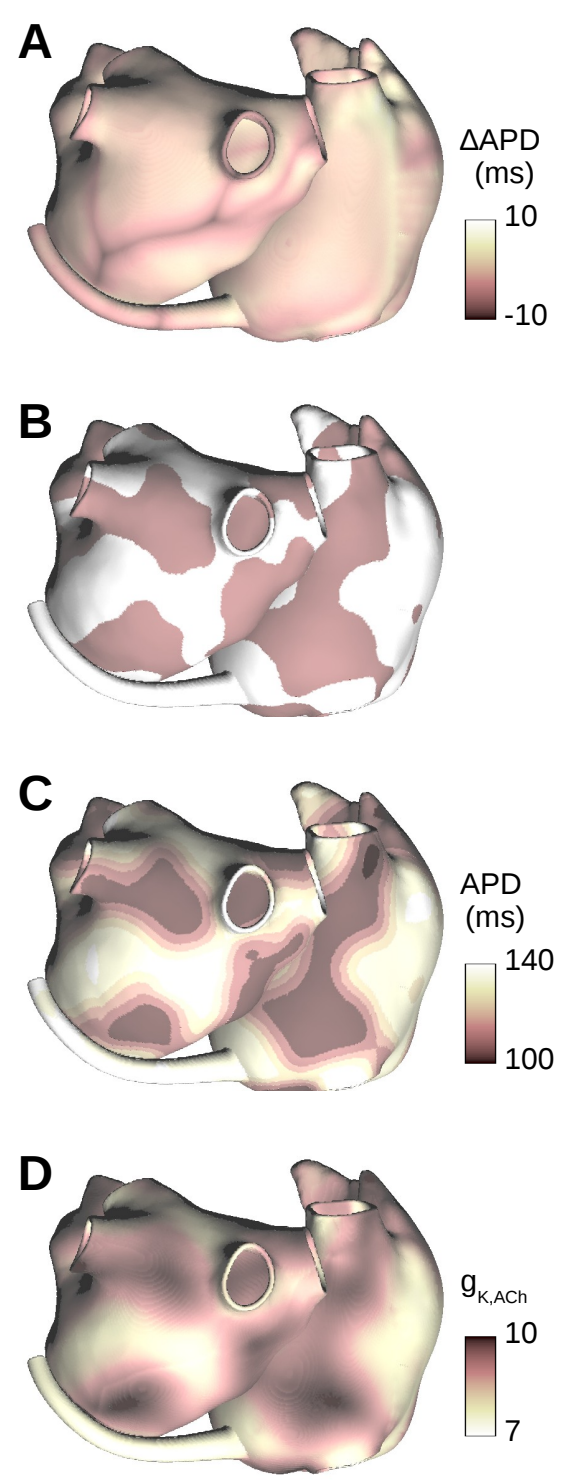

Figure 1. Example of inverse solution in an atrial model with longitudinal conductivity of $4 \mathrm{mS} / \mathrm{cm}$. (A) APD dispersion in the uniform case. (B) Random patchy regions. (C) Generated APD distribution with a maximal gradient of $2 \mathrm{~ms} / \mathrm{mm}$. (D) Solution to the inverse problem after ten iterations. The spatial map of the repolarization parameter $g_{\mathrm{K}, \mathrm{ACh}}$ is shown.

where the functions $\alpha^{-1}$ and $\alpha^{\prime}$ are applied element-wise and 'diag' transforms a vector into a diagonal matrix.

\subsection{Example in an atrial model}

The algorithm was tested in a simple model of the atria with rule-based fiber orientation [6]. Because the tissue space constant affects the spatial variations in APD $[1,3$, 
4], three cases with different conduction properties were simulated: the longitudinal/transverse conductivities were set to $2 / 0.5 \mathrm{mS} / \mathrm{cm}, 4 / 1 \mathrm{mS} / \mathrm{cm}$ or $9 / 3 \mathrm{mS} / \mathrm{cm}$, the latter configuration representing the baseline values in [6].

The Courtemanche-Ramirez-Nattel membrane kinetics was used, in which an $\mathrm{I}_{\mathrm{K}, \mathrm{ACh}}$ current was added. The formulation was the same as Kneller et al. [7], except that the pre-factor involving ACh was replaced by a conductance $g_{\mathrm{K}, \mathrm{ACh}}$. This parameter was taken as the control parameter $k$ responsible for APD variations. The range $\left[k_{\min }, k_{\max }\right]$ was set to $[0,15]$ for the estimation of the function $\alpha(k)$.

Given a spatial distribution of $k=g_{\mathrm{K}, \mathrm{ACh}}$, normal propagation from the sinus node was simulated. Since $\mathrm{I}_{\mathrm{K}, \mathrm{ACh}}$ can considerably reduce rate adaptation, only one beat was simulated. APD was measured at every node using a threshold at $-70 \mathrm{mV}$. This procedure constituted an implementation of the function $\mathbf{a}=\mathbf{a}_{\text {forw }}(\mathbf{k} ; \mathbf{G})$.

\subsection{Generation of APD maps}

Generation of target APD maps was based on random distributions of patches [8]. Four realizations were generated; one is shown on Fig. 1B. This defined a map $u_{0}(\mathbf{x})$ such that $u_{0}=0$ inside the colored patches and $u_{0}=1$ outside.

In order to control the gradient, a Gaussian spatial filter was applied by solving an isotropic diffusion equation $\partial u / \partial t=\Delta u$ with $u(\mathbf{x}, 0)=u_{0}(\mathbf{x})$ and no-flux boundary condition. The simulation code designed for the monodomain equation was used to solve the diffusion equation on the same grid. Analytical calculations in 1D show that the solution at time $T=\lambda^{2} / 2$ provides a map in the range $[0,1]$ with maximum gradient of approximately $(\sqrt{2 \pi} \lambda)^{-1}$. Accordingly, the target APD map was set to $a(\mathbf{x})=a_{0}+\Delta a \cdot u(\mathbf{x}, T)$, where $a_{0}=105 \mathrm{~ms}$, $\Delta a=30 \mathrm{~ms}$ and $T=\Delta a^{2} /\left(4 \pi \gamma^{2}\right)$. The gradient parameter $\gamma$ was $2,3.5$ or $5 \mathrm{~ms} / \mathrm{mm}$.

Boundary effects and wave front collisions also affect the APD [9]. A correction was applied to the generated maps to reduce overfitting in these regions. Otherwise, the algorithm would try to "cancel" these natural variations. Normal propagation was simulated in a uniform tissue with mean APD $=120 \mathrm{~ms}$ and the resulting APD variations were added to the target APD map. This assumes that the parameter $k$ does not significantly affect depolarization. Mathematically,

$$
\begin{aligned}
\mathbf{a}_{\text {unif }} & =\mathbf{a}_{\text {forw }}\left(\alpha^{-1}\left(a_{0}+\Delta a / 2\right) ; \mathbf{G}\right) \\
\mathbf{a}_{\text {target }} & =\mathbf{a}(\mathbf{x})+\mathbf{a}_{\text {unif }}(\mathbf{x})-\overline{\mathbf{a}_{\text {unif }}(\mathbf{x})}
\end{aligned}
$$

where the bar denotes the mean. Figure 1A displays an example of the map $\triangle \mathrm{APD}=\mathbf{a}_{\text {unif }}(\mathbf{x})-\overline{\mathbf{a}_{\text {unif }}(\mathbf{x})}$.

\subsection{Algorithm validation}

The algorithm for parameter estimation was tested on four realizations of the patchy heterogeneity pattern, three APD gradients, and three conduction properties, which makes 36 cases in total. Ten iterations of the algorithm were computed in each case.

\section{Results}

Figure 1 shows an example of inverse solution obtained using our algorithm. From a target APD map (Fig. 1C) that took into account the correction for collision and boundary effects (Fig. 1A), the spatial distribution of the repolarization parameter $g_{\mathrm{K}, \mathrm{ACh}}$ was reconstructed. Note that thanks to the correction, the resulting map (Fig. 1D) does not seem to overcorrect the APD at the locations of wave collisions (Fig. 1A). This parameter distribution may therefore be used for other propagation patterns.

The convergence of the iterations is summarized in Fig. 2. The error of the initial estimate ranged from 3 to $13 \mathrm{~ms}$. The initial estimate was close enough to the solution to ensure convergence of the Newton method provided that a solution existed. The first few iterations considerably reduced the error. Convergence was slower when tissue conductivity was higher and when the gradient was steeper.

To give some perspective on how steep these target gradients are, a cable with the same conductivity and discretization properties as the atrial model was simulated. The parameter $g_{\mathrm{K}, \mathrm{ACh}}$ was set to 9.5 (which corresponds to APD $=105 \mathrm{~ms}$ ) in the first half of the cable and 7 in the second half $(\mathrm{APD}=135 \mathrm{~ms})$. The resulting maximal APD gradient was $5.42 \mathrm{~ms} / \mathrm{mm}$ (for a conductivity of $2 \mathrm{mS} / \mathrm{cm}$ ), $3.83 \mathrm{~ms} / \mathrm{mm}(4 \mathrm{mS} / \mathrm{cm})$ and $2.55 \mathrm{~ms} / \mathrm{mm}(9 \mathrm{mS} / \mathrm{cm})$. This could arguably be considered to be the steepest physiological gradient (although in a cable the effect of anisotropy is ignored).

\section{Discussion}

Our algorithm enables the determination of the spatial distribution of a membrane kinetics parameter assuming that we know: (1) the geometry and the conduction properties, (2) the APD map during normal rhythm, and (3) which single parameter is the cause of APD dispersion. If the APD map at two different heart rates was available, the distribution of two parameters might be determined, but that would require an extension of the algorithm. In contrast, the method by Defauw et al. is less accurate but directly estimates the intrinsic APD without hypothesis about the ionic basis of APD variations [4].

In the test cases, the initial estimate (ignoring coupling) was reasonably good but systematically underestimated 

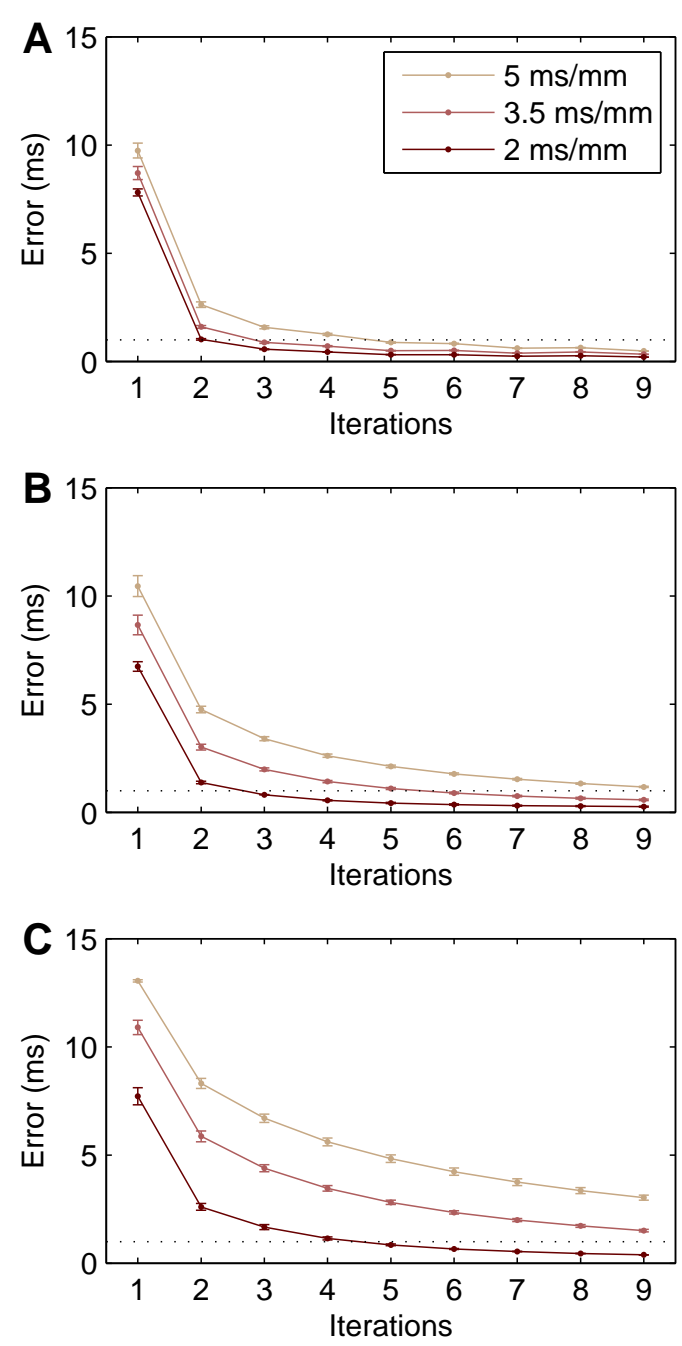

Figure 2. Error computed as the 99th percentile of the absolute error on the APD map along the iterations for three different maximal APD gradients: 2, 3.5 and $5 \mathrm{~ms} / \mathrm{mm}$. The longitudinal conductivity is $2 \mathrm{mS} / \mathrm{cm}$ (A), $4 \mathrm{~cm} / \mathrm{cm}$ (B) and $9 \mathrm{mS} / \mathrm{cm}(\mathrm{C})$. The error bars represent mean \pm standard deviation over 4 realizations of the heterogeneity distribution. The horizontal dotted line shows the 1-ms tolerance.

APD gradients. A few iterations (2 to 5) were sufficient to decrease the error below $1 \mathrm{~ms}$ provided that the maximum gradient was small. When the target maximum gradient was larger than the steepest physiological gradient obtained in a cable (discontinuity in the parameter), convergence was slower (e.g. $5 \mathrm{~ms} / \mathrm{mm}$ in Fig. 2B-C). An indication of non-existence of the solution is when the algorithm "hits the bounds" for the parameter $k$ (e.g. $k=0$ : a negative value would be needed to create a steeper gradient). In that case, the parameter profile converges but the error tends to zero only in regions of not-too-steep gradients.

\section{Conclusion}

APD heterogeneity creates a substrate for arrhythmias that can be investigated in computer models. To facilitate the design of such simulation studies, our algorithm provides a tool to automatically generate arrhythmogenic substrates with controllable repolarization gradients.

\section{Acknowledgements}

This work was supported by the Natural Sciences and Engineering Research Council of Canada (grant RGPIN2015-05658) and by the Mitacs Globalink program.

\section{References}

[1] Lesh MD, Pring M, Spear JF. Cellular uncoupling can unmask dispersion of action potential duration in ventricular myocardium. A computer modeling study. Circulation Research 1989;65(5):1426-1440.

[2] Joyner R. Modulation of repolarization by electrotonic interactions. Japanese heart journal 1986;27:167-183.

[3] Sampson KJ, Henriquez CS. Electrotonic influences on action potential duration dispersion in small hearts: a simulation study. American Journal of Physiology Heart and Circulatory Physiology 2005;289(1):H350-H360.

[4] Defauw A, Kazbanov IV, Dierckx H, Dawyndt P, Panfilov AV. Action potential duration heterogeneity of cardiac tissue can be evaluated from cell properties using Gaussian Green's function approach. PLoS ONE 2013;8(11):e79607.

[5] Jacquemet V. A simple analytical model of action potential duration profile in electrotonically-coupled cells. Math Biosci 2016;272:92-9.

[6] Jacquemet V. Modeling left and right atrial contributions to the ECG: A dipole-current source approach. Comput Biol Med 2015;65:192-9.

[7] Kneller J, Zou R, Vigmond EJ, Wang Z, Leon LJ, Nattel S. Cholinergic atrial fibrillation in a computer model of a twodimensional sheet of canine atrial cells with realistic ionic properties. Circulation research 2002;90(9):e73-e87.

[8] Jacquemet V, Virag N, Ihara Z, Dang L, Blanc O, Zozor S, Vesin JM, Kappenberger L, Henriquez C. Study of unipolar electrogram morphology in a computer model of atrial fibrillation. J Cardiovasc Electrophysiol 2003;14(10 Suppl):S172-9.

[9] Cherry EM, Fenton FH. Effects of boundaries and geometry on the spatial distribution of action potential duration in cardiac tissue. Journal of theoretical biology 2011;285(1):164176.

Address for correspondence:

Vincent Jacquemet

Hôpital du Sacré-Cœur de Montréal, Centre de Recherche

5400 boul. Gouin Ouest

Montreal (Québec) Canada H4J 1C5

vincent. jacquemet @umontreal.ca 